Urge capacitar a los gobiernos locales para la intersectorialidad por la salud: un policy brief

\title{
Capacity building for intersectoral action for health by local governments is needed: a policy brief
}

\author{
https://doi.org/10.23938/ASSN.0917
}

\author{
N. Hernantes ${ }^{1,2,3}$, E. Bermejo-Martins ${ }^{2,3,4}$, M.J. Pumar-Méndez ${ }^{2,4,5}$, O. López-Dicastillo ${ }^{2,4,5}$, \\ A. Iriarte-Roteta ${ }^{2,3,5,6}$, S. Fernández-Unanue ${ }^{1}$, A. Mujika ${ }^{2,7}$
}

\section{Sr. Editor:}

La salud es contexto-dependiente y el impacto que los gobiernos locales tienen en la misma es ineludible ${ }^{1}$. Por ello, cada municipio debe tener objetivos específicos que respondan a sus necesidades concre$\operatorname{tas}^{2}$. Y es que resulta evidente que la salud y el bienestar de la población no suponen exclusivamente la ausencia de la enfermedad, sino también el bienestar social, emocional y cultural de las personas en su propio entorno; y en ese entorno, cada uno debe ser capaz de alcanzar su máximo potencial como ser humano, contribuyendo así al bienestar de toda su comunidad ${ }^{3}$.

La salud depende de múltiples factores que están fuera del control de las personas y del sistema sanitario ${ }^{4}$. Esos factores, conocidos como determinantes sociales de la salud (DSS), suponen el 75\% del riesgo que las personas tienen de enfermar, dependiendo así su salud de las condiciones de vida que les rodean y no tanto del sistema sanitario ${ }^{5}$.

Los ayuntamientos, por su cercanía a la comunidad y la accesibilidad de los ciudadanos al mismo, gozan de una posición privilegiada para modificar los DSS. Pueden identificar las necesidades de salud e intervenir desde múltiples sectores, fomentando que la opción saludable sea la más fácil de escoger por parte de la población ${ }^{6,7}$.

A pesar de los avances teóricos en relación a enfoques que integran la perspectiva de salud en todos los sectores, tales como

\section{An. Sist. Sanit. Navar. 2020; 43 (3): 429-434}

1. Ayuntamiento de Donostia-San Sebastián. Dirección de Salud y Medio Ambiente. Departamento de Salud Pública. Sección de Promoción de la Salud. Donostia-San Sebastián.

2. Grupo de Investigación ImPuLS. Pamplona.

3. Departamento de Enfermería Comunitaria y Materno-Infantil. Facultad de Enfermería. Universidad de Navarra. Pamplona.

4. Instituto de Investigación Sanitaria de Navarra (IdiSNA). Pamplona.

5. Departamento de Ciencias de la Salud. Facultad de Ciencias de la Salud. Universidad Pública de Navarra. Pamplona.

6. Servicio Navarro de Salud - Osasunbidea. Pamplona.

7. Facultad de Medicina y Enfermería. Universidad del País Vasco. Donostia-San Sebastián.

\section{Correspondencia:}

Elena Bermejo-Martins

Departamento de Enfermería Comunitaria y

Materno-Infantil

Facultad de Enfermería

Universidad de Navarra

C/Irunlarrea, 1

31008 Pamplona (Navarra, España)

E-mail: ebermejo@unav.es

Recepción: 11/09/2020

Aceptación definitiva: 24/09/2020 
la Salud en Todas las Políticas (SeTP), el enfoque de Todo el Gobierno y la Gobernanza por la salud, su implementación real continúa siendo limitada. Esto es así, especialmente, para la colaboración intersectorial, que constituye un catalizador fundamental en dichos enfoques. Avanzar en este ámbito resulta fundamental para responder a la necesidad de integrar la salud como un eje trasversal en los gobiernos locales.

La adopción de este tipo de enfoques que pretenden integrar la perspectiva de salud, colaborar entre los sectores bajo un objetivo común en términos de salud y hacer de la salud un eje transversal en los ayuntamientos, no solo requiere el compromiso político, sino el convencimiento de toda la estructura administrativa responsable de ejecutar las decisiones políticas ${ }^{8}$.

Son múltiples los elementos que condicionan esta situación como, por ejemplo, contar con una legislación que fomente la integración de la perspectiva de salud en todas las áreas ${ }^{7}$ o la existencia de programas o estrategias del gobierno nacional coherentes con la perspectiva de los DSS ${ }^{9}$.

Por otro lado, la histórica tendencia natural al trabajo en silos supone una clara barrera; además, los sectores de los gobiernos locales tienen una estructura muy verticalizada, con sus propios directores, presupuestos, objetivos, resultados, lenguaje y tecnicismos ${ }^{10}$.

A ello se le suma una concepción reduccionista de la salud, bajo el predominio de una perspectiva biomédica ${ }^{11}$. Esta visión desatiende los impactos sociales y medioambientales y deposita la responsabilidad de los estilos de vida en las personas; así, paradójicamente, la salud pública fomenta en ocasiones la culpabilización de las víctimas (victim blaming) ${ }^{12}$.

El predominio de un enfoque basado en la enfermedad unido al trabajo en silos, conllevan una falta de lenguaje común que dificulta la comunicación entre los sectores para abordar cuestiones relacionadas con la salud ${ }^{11}$. Así, las distintas áreas de un gobierno local tampoco llegan a ser conscientes del impacto que estos tienen en la salud y el bienestar de la población y atribuyen generalmente estas competencias a los departamentos de salud pública, o bien al sistema sanitario ${ }^{13}$. En este escenario, los sectores no-salud consideran que abordar cuestiones de salud supone una tarea ex$\operatorname{tra}^{6,7}$.

Todo esto dificulta una visión compartida a través de los sectores y el establecimiento de objetivos comunes que precisen colaboración para su consecución ${ }^{7,14}$. Además, se ha evidenciado una falta de compromiso $^{11}$, responsabilidad política y rendición de cuentas en cuestiones de sa$\operatorname{lud}^{7}$. En términos generales, existe una falta de capacidad para abordar la promoción de la salud desde un enfoque intersectorial en los gobiernos locales ${ }^{9,15,16}$.

A pesar de todo lo expuesto, el contexto español es favorable para avanzar en este ámbito. La Ley 33/2011, de 4 de octubre, General de Salud Pública ofrece un marco legal que sustenta la adopción del enfoque de SeTP; así queda reflejado también en la Estrategia de Promoción de la Salud y Prevención en el Sistema Nacional de Salud, entre otros. La importancia de la intersectorialidad en lo local, destaca en la guía de implementación local de la estrategia, publicada con el fin de acercar y potenciar la implementación de la estrategia en los contextos locales. Asimismo, deriva de La ley $27 / 2013$, de racionalización y sostenibilidad de la Administración Local, que atribuye a los municipios, en su artículo 25, competencias que tienen un impacto en la salud de la población; materias como urbanismo, medioambiente urbano, infraestructura viaria y equipamiento, policía local, protección civil, tráfico, y movilidad.

Además, aunque el mayor número de experiencias publicadas en España son a nivel provincial ${ }^{17}$, cada vez son más los municipios que cuentan con experiencias exitosas en este ámbito ${ }^{18}$.

Para avanzar en el establecimiento de la salud como eje trasversal en los gobiernos locales las autoras proponen tres recomendaciones. La primera tiene que ver con la existencia de presupuesto destinado a este fin; la segunda está orientada a la institucionalización del uso de herramientas para medir impacto en salud, y la tercera propone trabajar la creación de capacidad para 
asentar las bases esenciales y ofrecer un escenario en el que las acciones recomendadas puedan integrarse y actuar sinérgicamente:

1. La literatura destaca la necesidad de crear partidas presupuestarias específicas provenientes tanto de los presupuestos de los gobiernos locales, como de los gobiernos centrales que posibiliten el trabajo en este ámbito ${ }^{7,19}$. Respecto a los primeros, si bien incluir una partida para abordar cuestiones de salud en cada uno de los departamentos puede llegar a ser complejo, se recomienda que las áreas que están en la cabeza de la dirección del gobierno local creen partidas presupuestarias transversales bajo las que se pueda integrar la salud. Además, estas deben ir acompañadas de los oportunos mecanismos de rendición de cuentas, tal y como ya se hace en algunos países nórdicos ${ }^{20,21}$.

2. También se recomienda, para estimular la colaboración, la institucionalización de herramientas que ayuden a medir el impacto en salud? ${ }^{7}$. Un ejemplo es la herramienta de Evaluación del Impacto en Salud (EIS); se ha descrito que su empleo fomenta la concienciación sobre los DSS a través de los distintos sectores y existen experiencias exitosas de su uso ${ }^{22,23}$. Sin embargo, en algunos contextos ha supuesto una barrera más que un facilitador debido a su diseño tecnocrático y racional a la hora de tomar decisiones ${ }^{24}$. También está disponible la herramienta Urban HEART ${ }^{25}$, empleada de forma exitosa en Barcelona ${ }^{26}$, que los gobiernos locales pueden utilizar para medir y monitorizar la equidad en salud y las intervenciones relevantes en distintos sectores ${ }^{27}$. Se tiene la experiencia de un gobierno local en Dinamarca que creó su propia herramienta para integrar las cuestiones de salud en todos los sectores, denominada Pitch Template, y que ha tenido buenos resultados ${ }^{14}$. Si bien existe la posibilidad de escoger entre distintas herramientas, cada gobierno local deberá emplear aquella que más se ajuste a sus necesidades y funcionamiento organizacional. Y en caso de no considerar ninguna de las alternativas adecuadas se podría involucrar a todos los sectores en el desa- rrollo de una herramienta simple y amigable para integrar las cuestiones de salud en todos ellos ${ }^{27}$.

3. Una última recomendación, fundamental bajo el criterio de los autores, es trabajar en la creación de capacidad (capacity building) para avanzar en el abordaje de la salud de forma eficiente desde los gobiernos locales. La creación de capacidad se entiende como un proceso que comprende el desarrollo del conocimiento, las habilidades, el compromiso, las estructuras, los recursos, los sistemas y el liderazgo para promover la salud de forma efectiva ${ }^{28}$. Este marco ofrece un escenario en el cual integrar todas las acciones recomendadas y descritas en la literatura de una forma sinérgica. Trabajar la capacidad resulta condición sine qua non para poder adoptar e implementar enfoques que apuesten por la integración de la perspectiva de salud en todas las áreas que componen un gobierno local y avanzar así en la promoción la salud de los ciudadanos de una manera sostenible y eficaz ${ }^{29,30}$. Además, se debe poner especial énfasis en los trabajadores de los gobiernos locales, de los cuales se espera que tengan conocimiento, aptitudes y competencias para poder abordar temas de salud que tienen un impacto directo sobre las personas ${ }^{15}$.

Junto a estas recomendaciones, las autoras proponen que sea el sector salud quien asuma el liderazgo en este proceso. Contar con un referente claro para avanzar en la integración de la perspectiva de la salud en todas las áreas es el apunte más frecuentemente realizado en la literatura, unido a la consolidación de un grupo de trabajo intersectorial ${ }^{6,9,11,14-16,31}$. El sector salud puede acompañar los procesos de implementación de enfoques como la SeTP y la creación de capacidad en los sectores. Además, la función de ejercer como punto de anclaje entre todos ellos resulta fundamental, destacando en este sentido figuras como la del bróker de la salud descritas en la evidencia ${ }^{32,33}$.

El sector salud debe trabajar en capacitar a los sectores concienciándoles del impacto que tienen en la salud de la población, fomentando su compromiso por 
el abordaje de la salud y avanzando en la rendición de cuentas en estos términos. Además, es preciso que se reconozca el trabajo que ya puedan estar realizando (aunque quizá no identificado como tal) con implicaciones positivas para la salud de la población. Este último punto es clave para fomentar el trabajo por la salud dentro de estructuras ya consolidadas, sin que las cuestiones de salud tengan que suponer necesariamente una tarea extra siempre. Este último elemento, el aprovechamiento de las estructuras y actividades contextuales, es fundamental en el proceso de creación de capacidad $^{34}$.

En conclusión, los enfoques que integran la salud en todos los sectores y sus políticas están llamados a la acción; sin embargo, la colaboración entre los sectores y el convencimiento de la parte política y de la estructura administrativa continúa siendo la principal barrera para poder implementarlos. En muchos casos, fomentar la colaboración puede requerir mecanismos de financiación e incentivos, o institucionalizar herramientas que evalúen las consecuencias en salud de las políticas y decisiones de todos los sectores. Las autoras, como paso previo a estas medidas, recomiendan la capacitación de los sectores para fomentar una visión compartida en torno a la salud como elemento esencial para establecer objetivos comunes. Esta capacitación debe ser liderada por el sector salud. Ello permitirá avanzar en el establecimiento de la salud como un eje trasversal en los gobiernos locales para promover la salud de las poblaciones sobre las cuales gobiernan.

\section{Agradecimientos:}

A la Asociación de Amigos de la Universidad de Navarra y al Departamento de Educación del Gobierno de Navarra por las becas predoctorales concedidas. Al grupo Health Promotion in Settings, de la Universidad del Sudeste de Noruega, por las discusiones científicas y reflexiones compartidas durante la estancia en dicha universidad.

\section{BIBLIOGRAFÍA}

1. World Health Organization. Addresing the social determinants of health: the urban dimension and the role of local government. Denmark 2012. https://www.euro.who.int/_ data/assets/pdf_file/0005/166136/UrbanDimensions.pdf

2. Jansson EV, TillgRen PE. Health promotion at local level: a case study of content, organization and development in four Swedish municipalities. BMC Public Health 2010; 10: 455. https://doi.org/10.1186/1471-2458-10-455

3. The Health Foundation. What makes us healthy? An introduction to the social determinants of health. 2018: 62. Available from: http://www.health.org.uk/sites/health/files/ What-makes-us-healthy-quick-guide.pdf

4. CSDH. Closing the gap in a generation: health equity through action on the social determinants of health. Final Report of the Commission on Social Determinants of Health. Geneva: World Health Organization, 2008. https://apps.who.int/iris/bitstream/handle/10665/43943/9789241563703_eng.pdf

5. Keshavarz Mohammadi $\mathrm{N}$, Taheri $\mathrm{F}$, Motallebi M, Yazdanpanah A, Khosravi Y, Borhani JebeLI $\mathrm{M}$ et al. Development of a national conceptual framework and measuring tool for Organisational Social Responsibility and Accountability for Health (OSRAH). Glob Health Promot 2019; 27: 17-25. https://doi. org/10.1177/1757975918789346

6. Van Vliet-Brown CE, Shahram S, OelKe ND. Health in All Policies utilization by municipal governments: scoping review. Health Promot Int 2018; 33: 713-722. https://doi.org/10.1093/ heapro/dax008

7. Guglielmin M, Muntaner C, O'Campo P, ShankarDASS K. A scoping review of the implementation of health in all policies at the local level. Health Policy (New York) 2018; 122: 284-292. https://doi.org/10.1016/j.healthpol.2017.12.005

8. CAREY G, Friel S. Understanding the role of public administration in implementing action on the social determinants of health and health inequities. Int $\mathrm{J}$ Heal Policy Manag 2015; 4: 795-798. https://doi.org/10.15171/ ijhpm.2015.185

9. Fosse E, Helgesen MK, Hagen S, Torp S. Addressing the social determinants of health at the local level: opportunities and challenges. Scand J Public Health 2018; 46 (Suppl 20): 47-52. https://doi. org/10.1177/1403494817743896 
10. Holt DH, Carey G, Rod MH. Time to dismiss the idea of a structural fix within government? An analysis of intersectoral action for health in Danish municipalities. Scand J Public Health 2018; 46 (Suppl 22): 48-57. https:// doi.org/10.1177/1403494818765705

11. Scheele CE, Little I, Diderichsen F. Governing health equity in Scandinavian municipalities: the inter-sectorial challenge. Scand J Public Health 2018; 46: 57-67. https://doi. org/10.1177/1403494816685538

12. Jancey J, Barnett L, Smith J, Binns C, Howat $\mathrm{P}$. We need a comprehensive approach to health promotion. Heal Promot J Aust 2016; 27: 1-3. https://doi.org/10.1071/hev27n1_ed

13. Freiler A, Muntaner C, Shankardass K, Mah CL, Molnar A, Renahy E et al. Glossary for the implementation of Health in All Policies (HiAP). J Epidemiol Community Health 2013; 67: 1068-1072. https://doi.org/10.1136/jech-2013202731

14. Holt DH, Rod MH, WaLdorfF SB, TuøRnhøנThomsen T. Elusive implementation: an ethnographic study of intersectoral policymaking for health. BMC Health Serv Res 2018; 18: 54. https://doi.org/10.1186/s12913-018-2864-9

15. Weiss D, Lillefjell M, Magnus E. Facilitators for the development and implementation of health promoting policy and programs - a scoping review at the local community level. BMC Public Health 2016; 16: 140. https://doi. org/10.1186/s12889-016-2811-9

16. SynNEvåg ES, AmdAM R, Fosse E. Intersectoral planning for public health: dilemmas and challenges. Int $\mathrm{J}$ Heal Policy Manag 2018; 7: 982-992. https://doi.org/10.15171/ ijhpm.2018.59

17. Mateu i Serra A. Salud en Todas las Políticas e intersectorialidad en la promoción de la salud: el Plan Interdepartamental de Salud Pública (PINSAP) de Cataluña. Med Clin (Barc) 2015; 145: 34-37. https://doi.org/10.1016/ s0025-7753(15)30036-1

18. Borrell C, Pasarín Mi, Díez E, Pérez K, MalMusi D, PÉrez $\mathrm{G}$ et al. Las desigualdades en salud como prioridad política en Barcelona. Gac Sanit 2020; 34: 69-76. https://doi. org/10.1016/j.gaceta.2019.04.004

19. McGuire F, Vijayasingham L, Vassall A, Small R, Webв D, Guthrie T et al. Financing intersectoral action for health: a systematic review of co-financing models. Global Health 2019; 15 : 86. https://doi.org/10.1186/s12992-019-05137

20. MelKas T. Health in all policies as a priority in Finnish health policy: a case study on national health policy development. Scand J Pu- blic Health 2013; 41(Suppl 11): 3-28. https:// doi.org/10.1177/1403494812472296

21. Hagen S, Torp S, Helgesen M, Fosse E. Promoting health by addressing living conditions in Norwegian municipalities. Health Promot Int 2016; 32: 977-987. https://doi.org/10.1093/ heapro/daw052

22. Vela-Ríos J, Rodríguez-Rasero FJ, Moya-Ruano LA, Candau-Bejarano A, Ruiz-Fernández J. Institucionalización de la evaluación del impacto en la salud en Andalucía. Gac Sanit 2016; 30: 81-84. https://doi.org/10.1016/j.gaceta.2015.08.009

23. Morteruel M. Valoración de la efectividad y sus determinantes de las Evaluaciones de Impacto en Salud en el estado español. Tesis doctoral: Universidad del País Vasco, 2017. https://www.ehu.eus/ documents/3638427/7319509/Tesis-MaiteMorteruel.pdf

24. Holt DH, Frohlich KL, Tuørnhøj-Thomsen T, ClaVIER C. Intersectoriality in Danish municipalities: corrupting the social determinants of health? Health Promot Int 2017; 32: 881-890. https://doi.org/10.1093/heapro/daw020

25. Organización Mundial de la Salud, Centro OMS para el Desarrollo Sanitario. Urban HEART : instrumento de evaluación y respuesta en materia de equidad sanitaria en los medios urbanos. Kobe: World Health Organization, 2010. https://apps.who.int/iris/ handle/10665/79063

26. Novoa AM, Pérez G, Espelt A, Echave C, de OlaLla PG, CAlvo MJ et al. The experience of implementing urban HEART Barcelona: a tool for action. J Urban Health 2018; 95: 647-661. https://doi.org/10.1007/s11524-017-0194-6.

27. Larsen M, Rantala R, Koudenburg OA, Gulis G. Intersectoral action for health: the experience of a Danish municipality. Scand J Public Health 2014; 42: 649-657. https://doi. org/10.1177/1403494814544397

28. World Health Organization. Health Promotion Glossary. Geneva: World Health Organization, 1998. https://www.who.int/healthpromotion/about/HPR\%20Glossary\%201998. pdf?ua

29. Von Heimburg D, HaKKebo B. Health and equity in all policies in local government: processes and outcomes in two Norwegian municipalities. Scand J Public Health 2017; 45 (Suppl 18): 68-76. https://doi. org/10.1177/1403494817705804

30. Bergeron K, Abdi S, DeCorby K, Mensah G, RemPEL B, MANSON H. Theories, models and frameworks used in capacity building interventions relevant to public health: a systematic 
review. BMC Public Health 2017; 17: 914. https://doi.org/10.1186/s12889-017-4919-y

31. Storm I, den Hertog F, van Oers H, Schuit AJ. How to improve collaboration between the public health sector and other policy sectors to reduce health inequalities? - A study in sixteen municipalities in the Netherlands. Int J Equity Health 2016; 15: 97. https://doi. org/10.1186/s12939-016-0384-y

32. van Rinsum CE, Gerards SMPL, Rutten GM, van DE GOOR IAM, KREMERS SPJ. Health brokers: how can they help deal with the wickedness of public health problems? Biomed Res Int. 2017. https://doi.org/10.1155/2017/1979153

33. Harting J, Kunst AE, Kwan A, Stronks K. A "health broker" role as a catalyst of change to promote health: an experiment in deprived Dutch neighbourhoods. Health Promot Int 2011; 26: 65-81. https://doi.org/10.1093/ heapro/daq069

34. New South Wales Health Department. A framework for building capacity to improve health. Gladesville: New South Wales Health Department, 2001. 